\title{
JEJUNO-JEJUNAL INTUSSUSCEPTION DUE TO LIPOMA
}

Shrestha S R*, Shah J N

\section{ABSTRACT}

Sixty eight year old lady from Kathmandu presented to Emergency Department, Patan Hospital with pain and abdomen distention associated with vomiting and constipation. Abdomen examination revealed a mass in infraumblical region. Emergency laparotomy for acute intestinal obstruction revealed jejuno-jejunal intussusception due to multiple lipoma of jejunum intestine. Resection anastomosis was done. Postoperative period was uneventful.

\section{Key Words: Intussusception, Intestinal obstruction, Jejunum, Lipoma.}

\section{INTRODUCTION}

Intussusception is a process in which a portion of the gut becomes invaginated within an immediately adjacent segment, invariably it is the proximal into distal bowel. ${ }^{1}$ This leads to obstruction of venous and lymphatic flow which may further progress to mesenteric artery obstruction and ischaemia of the bowel. Intussusception in adult is uncommon, approximately $5 \%$ of all intussusceptions and $1 \%$ of bowel obstructions. ${ }^{2}$

Small bowel obstruction due to enteroenteral intussusceptions like jejuno-jejunal, jejuno-ileal and ileo-ileal are usually associated underlying pathology, mainly some intraluminal growth. Small bowel lipomas are usually submucosal and do not produce symptoms until they reach $4 \mathrm{~cm}$. They may cause blood loss due to ulceration of the overlying mucosa in addition to intussusception.

\section{CASE REPORT}

A sixty eight year old lady, farmer from Kathmandu, presented to emergency department Patan Hospital with complaints of sudden continuous periumblical pain associated with vomiting, abdomen distension and constipation for 2 days. She was on medication for Chronic Obstructive Airway Disease (COPD) and Acid Peptic Disease (APD).

On examination, she was restless in bed. Her pulse was 90/ min, respiration 18/min, blood pressure 130/86 $\mathrm{mmHg}$. She was non-icteric. Respiratory system, cardiovascular system appeared normal. Abdomen was distended, mainly umbilical and suprapubic region. No visible peristalsis was seen. A tender, mobile mass was palpated in infraumblical region. Bowel sound was sluggish. Per rectal examination was normal.

\section{Investigations}

Leucocyte count, Haematocrit, Serum amylase, Serum creatinine, Sodium, Potassium and Abdomen X-ray were normal.

With provisional diagnosis of acute intestinal obstruction (? intussusception), patient was resuscitated in the emergency department and planned for surgery. Emergency laparotomy revealed jejuno-jejunal intussusception. Manual reduction was not tried due to patchy bowel necrosis. Mass resected, $8 \mathrm{~cm}$ from Ligament of Traits to $50 \mathrm{~cm}$ distally (fig. 1, 2) and anatomosed end to end in two layer. Postoperative period was uneventful. Histopathology - jejunal lipomas.

* Patan Hospital, Lalitpur, Kathmandu, Nepal.

Address for correspondence : Dr. Sita Ram Shrestha

Patan Hospital, Lalitpur, Kathmandu, Nepal

Email: drshrestha@yahoo.co.uk 


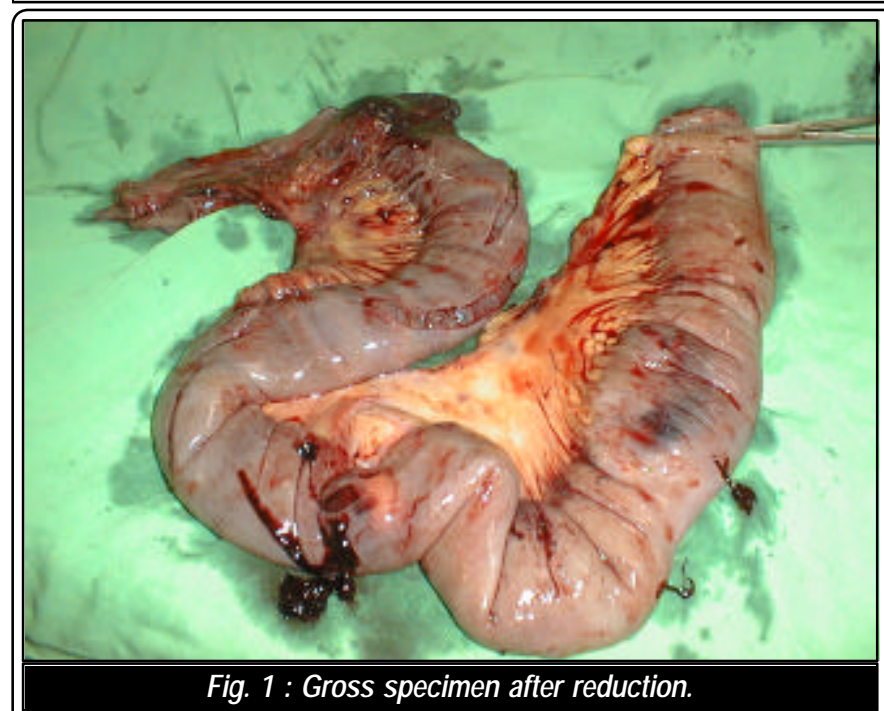

\section{DISCUSSION}

Intussusception is the disease of childhood, in which ileum (intussusceptum) telescopes into the colon (intussuscipiens) an ileocolic intussusception. During this period, there is usually no lead point and intussusception is thought to be due to a viral infection that results in either enlarged lymph node or bowel wall inflammation. Enteroenteric type of intussusception is rare in children.

In contrast, intussusception in adult is uncommon, approximately $5 \%$ of all intussusceptions and $1 \%$ of bowel obstructions $^{2}$ and usually caused by underlying pathology, malignant growth in colon and benign tumors in small bowel. This is the first case reported from Patan Hospital.

The pathogenesis an imbalance in the longitudinal forces along the intestinal wall, caused by a mass acting as a lead point or by a disorganized pattern of peristalsis. As a result, an area of the intestinal wall invaginates into the lumen, with the rest of the intestine following. The invaginating portion of the intestine is called intussusceptum, which completely invaginates into the receiving portion of the intestine known as intussuscipiens. This process continues and more areas follow, and allows to intussusceptum to proceed along the lumen of the intussuscipiens.

The mesentery of the intussusceptum is invaginated with the intestine, which leads to classic pathophysiologic process of any bowel obstruction.

Jejuno-jejunal intususception in adults may result due to lipoma, ${ }^{3,4}$ leimyoma, ${ }^{6}$ leiomyosarcoma, ${ }^{7}$ Denis tube insertion for ileus, ${ }^{8}$ melanomas, adenomatous polyps, after Roux-en-Y

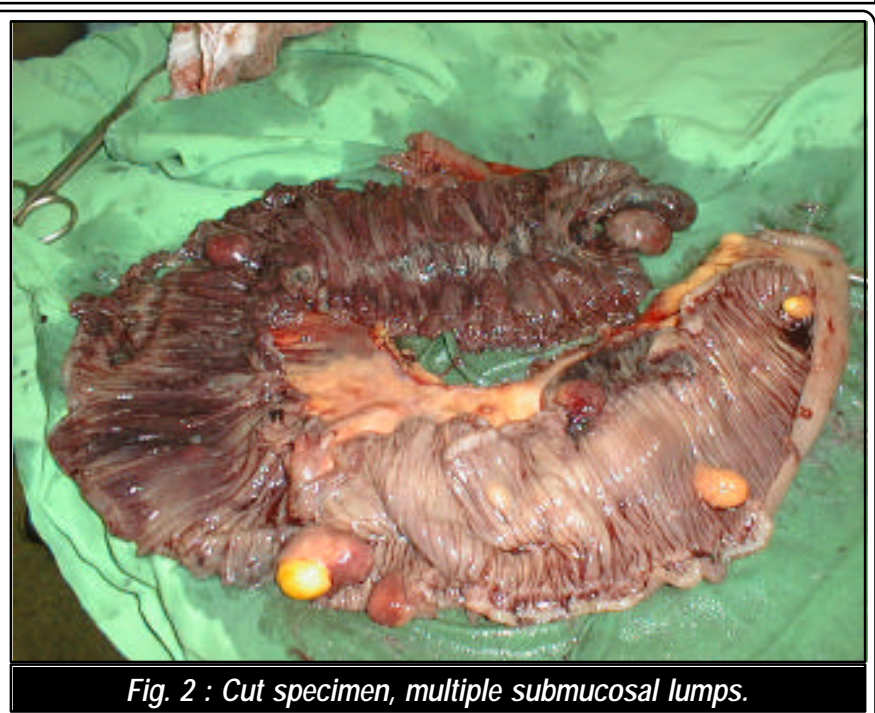

gastrojejunostomy, ${ }^{9}$ Trichobezoar with tail, ${ }^{10}$ and metastatic renal cell carcinoma. ${ }^{11}$ Lipoma may be sub-mucosal as in our case and as described by Cakirer, ${ }^{4}$ polypoid,${ }^{10}$ hemorrhagic. ${ }^{11}$

Clinical presentation of intussusception in adults in contrast to children tends to be more chronic or intermittent and include pain, constipation, weight loss or a palpable mass at physical examination. In children presentation is usually acute with colicky abdominal pain, vomiting, bloody stool that look like current jelly, and often a palpable mass. ${ }^{1}$

In many cases it is difficult to diagnose jejuno-jejunal intussusception on the basis of clinical presentation. Patient presenting with signs of intestinal obstruction and with abdominal mass should be suspected as a case of Intussusception. Jejuno-jejunal intussusception can be diagnosed with the help of ultra sonogram of abdomen, typically multilayered appearance consisting of the alternating hypoechoic concentric rings that represent alternating layers of mucosa, bowel wall, and mesenteric fat in cross section, ${ }^{3}$ plain X ray of abdomen shows dilated bowel, a paucity of bowel gas in the right lower quadrant, or a soft tissue mass that produces a concave defect in the air column of the colon, ${ }^{3} \mathrm{CT}$ findings include a target like or sausage like mass, depending on the angle of the beam relative to the intususception, in which the inner central area represents the invaginated intussusceptum that is surrounded by its mesenteric fat and associated vasculature, all of which are surrounded by the thick-walled intussuscipiens. ${ }^{3}$

In children, conservative or operative reduction of uncomplicated intususception is usually sufficient, but in adult resection is required to cure the underlying cause. 


REFERENCES
1. R.C.G. Russell, Norman S. Williams and Christopher
J.K.Bulstrode. Intestinal dostnuction. In Bailey and Love's Short
Practice of Surgery. 2000; 58, 1058-1070.

2. David M.Warshauer, MD and Joseph K. Lee, MD. Adult Intussususception Detected at CT or MR imaging: Clinical imaging correlation. Radiology. 1999; 212: 853-860

3. Ross GJ, Amilineni V. Case 26: Jejunojejunal Intussusception secondary to a Lipoma. Radiology 2000; 216 (3) : 727-30.

4. Cakirer $\mathrm{S}$, Sirvanci M, Duran C. Jejunojejunal intussusception secondary to Lipoma in an adult. Acta Radiol. 2002; 43 (2) : 196-7.

5. Unbano J, Serantes A, Hernandez L, Turgano F. Lipoma induced jejunojejunal intussusception: US and CT diagnosis. Abdo Imaging. 1996; $21(6): 522-4$.

6. Mansberg VJ, Mansberg G, Doust BD. Jejunojejunal intussusception secondary to Leiomyoma. Australas Radiology; 40 (1) : $72-4$.
7. Yasuda $\mathrm{H}$, Mine $\mathrm{T}$, Idehara A et al. A case of adult jejunojejunal intussusception with Leiomyosarcoma diagnosed by computed tomography. Nippon Shokakibyo Gakki Zasshi . 1989; 86 (12) : 2804-7.

8. Ophoff $\mathrm{K}$, Riesener KP, Buchin P, Troung S, Schumpelick V. Jejuno- jejunal invagination after Dennis tube insertion for ilusa rare compication. Chirurg. 1999; 70 (6) 721-3.

9. Loizou MC, Koundourskis, Kollias VD et al. Jejuno- jejunal intussusception after Roux-en-Y gastrojejunostomy: a rare cause of postoperative bowel obstruction in an adult. Case report. Eur J Surgery. 1994; 160 (8) : 451-2.

10. Weiss M, Danoff DM, Wood BP. Radiological case of the month. Jejunojejunal intussusception caused by a Trichobezoar with a tail. Arch Pediatr Adolesc Med. 1998; 152 (4) : 403-4.

11. Savoye-collet C, Thoumas D, and Dunet F et al. CT diagnosed jejunojejunal intussusception revealing metastatic renal cell carcinoma. JBR-BTR. 2001; 84.

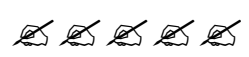

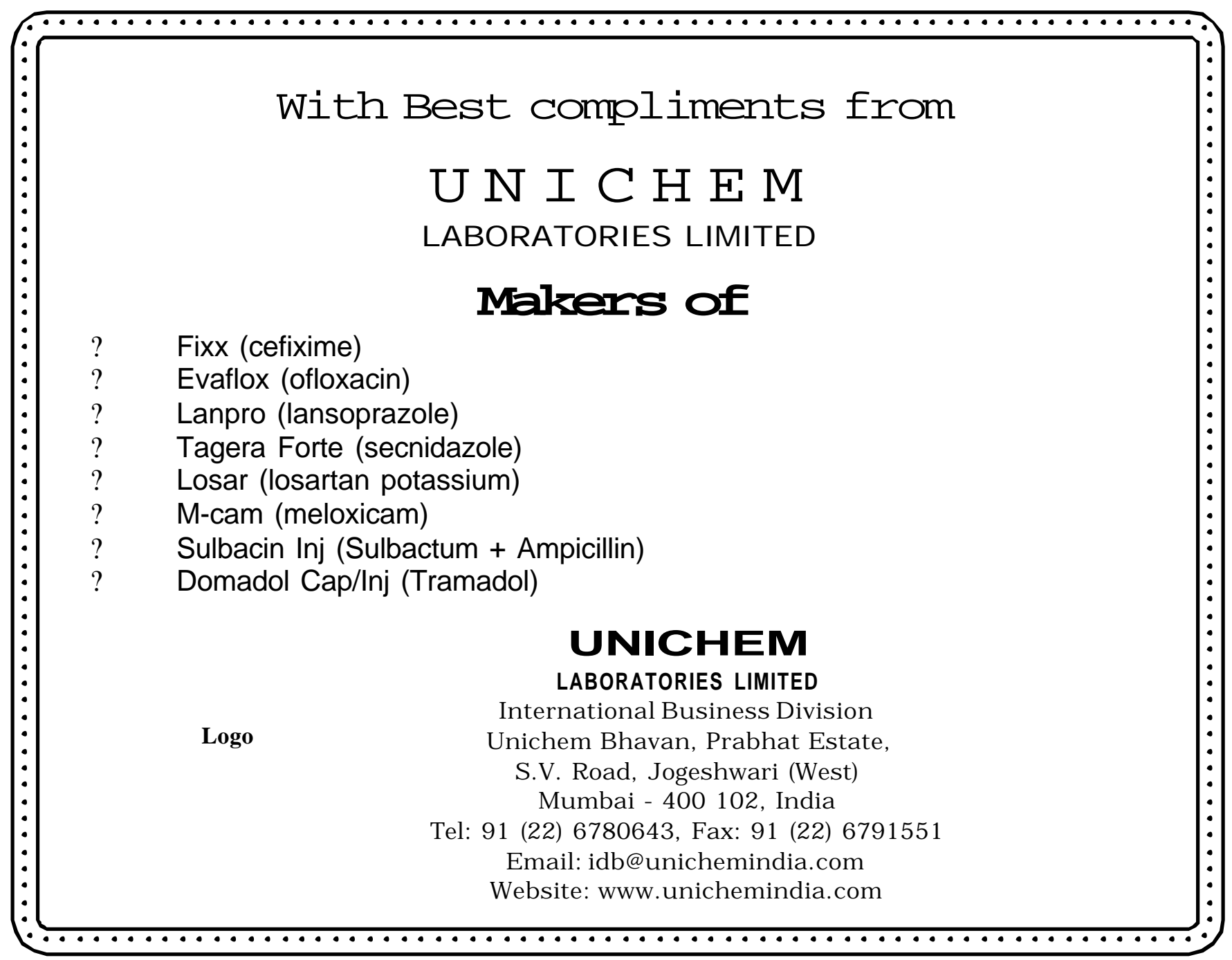

